\title{
QCD at colliders
}

\author{
Claudia Glasman* Universidad Autónoma de Madrid \\ Cantoblanco 28049, Madrid, Spain \\ E-mail: Claudia.qlasmanduam.es
}

Recent results on tests of QCD from a variety of experiments at HERA, TevaTron and LHC are reviewed. Measurements of jet, $W$ and $Z$ boson, photon and heavy-flavour production are presented. Besides their intrinsic interest, these measurements provide experimental constraints on Standard Model processes that constitute background to new physics and are used to extract values of the coupling of the strong interaction and to constrain the proton parton distribution functions. New developments in theoretical calculations relevant for the comparison to the measurements are also discussed.

XXVII International Workshop on Deep-Inelastic Scattering and Related Subjects - DIS2019 8-12 April, 2019

Torino, Italy

${ }^{*}$ Speaker. 


\section{Introduction}

Multi-jet QCD processes are dominant at hadron colliders. Besides their intrinsic interest, these processes represent usually a background to other processes and new physics. Thus, a good understanding of QCD is needed to extract new signatures.

The simplest way to study QCD is in $e^{+} e^{-}$colliders, like LEP, in which QCD enters only in the final state. At LEP, the study of jet production led to precise measurements of the strong coupling, $\alpha_{\mathrm{s}}$, and tests of colour dynamics. At the ep collider HERA, a simple hadronic environment provides well understood measurements of the proton structure and of $\alpha_{\mathrm{s}}$ and tests of the photon structure. The $p \bar{p}$ collider TevaTron provided measurements of $\alpha_{\mathrm{s}}$ and tests of the matrix elements and the proton structure.

All the knowledge acquired on QCD in all these previous experiments is now applied on the challenging measurements performed at LHC. The value for the coupling $\alpha_{\mathrm{s}}$, the parameterisations of the proton parton distribution functions (PDFs) and the partonic cross sections computed at the highest possible orders are essencial input ingredients for any theoretical prediction at LHC, such as the Higgs production cross section. For such prediction, a well understood gluon density at low $x$ is needed to calculate accurately the partonic luminosity. For the production of $W$ or $Z$ bosons, a good knowledge of the quark densities are needed. The coverage of the existent data from HERA and fixed-target experiments in the $Q^{2}-x$ plane is such that any particle with mass e.g. above $100 \mathrm{GeV}$ is produced now at LHC in a region of $x$ where there exist data and only the well-tested DGLAP evolution equations at high $Q^{2}$ are needed to compute the predictions.

Tests of QCD are performed at colliders using different observables, such as structure functions and heavy quark, jet and particle production. All these observables provide information on the proton parton densities, the fundamental parameters of the theory and test parton dynamics at the highest possible energies. It is also possible to try to understand non-perturbative effects from first principles and search for breakdowns of the evolution equations.

\section{Probing QCD at the highest energies and at the highest orders with jets}

Traditionally, perturbative QCD (pQCD) is studied by measuring jet cross sections, which can test QCD at the highest energies with highest rates. The CMS [U] and ATLAS [ [ ] Collaborations have measured the inclusive-jet cross section as a function of jet transverse momentum ( $\left.p_{\mathrm{T}}^{\text {jet }}\right)$ in different regions of jet rapidity $\left(y^{\text {jet }}\right)$ using the anti- $k_{\mathrm{t}}$ jet algorithm with different jet radii with Run II data at LHC with a centre-of-mass energy $(\sqrt{s})$ of $13 \mathrm{TeV}$. These measurements are sensitive to new physics at high scales and also can constrain the PDFs. The high precision measurements, shown in Fig. W, cover a region up to $4 \mathrm{TeV}$, and constitute the most stringent test of pQCD at the highest available energies so far. The next-to-leading-order (NLO) QCD calculations, including electroweak effects, give a reasonable description of the data. The next-to-next-to-leading-order (NNLO) are expected to provide a better description of the data with a significant reduction of the theoretical uncertainties. This is obtained up to a certain extent, but still ambiguities with the scale choice are present, as can be seen in Figs. W(c) and (d).

Recently, even higher orders in the perturbative expansion have become available. Jet production predictions in neutral-current (NC) and charged-current (CC) deep inelastic scattering (DIS) 
are available since 1995 at NLO. It took two decades to earn one more order and now, just a few years later, next-to-next-to-next-to-leading-order $\left(\mathrm{N}^{3} \mathrm{LO}\right)$ calculations are starting to become available for a limited selection of processes. The use of the Projection-to-Born (P2B) method, based on the available $\mathrm{N}^{3} \mathrm{LO}$ inclusive cross secions and NNLO differential cross sections, allows the computation of $\mathrm{N}^{3} \mathrm{LO}$ differential cross sections for jet production. Figure $\mathrm{W}(\mathrm{e})$ and (f) shows calculations at leading order (LO), NLO, NNLO and $\mathrm{N}^{3} \mathrm{LO}$ [目, 目] compared to ZEUS NC [B] and CC [ [ $⿴ 囗$ ] DIS jet cross sections as functions of $p_{\mathrm{T}}^{\text {jet }}$. The excellent description of the data as provided by the $\mathrm{N}^{3} \mathrm{LO}$ predictions is clearly seen; the improvement is significant in regions of phase space where the NNLO calculations fail to describe the data. In addition, the scale uncertainties are significantly reduced. These predictions constitute an important step towards a fully differential calculation of vector-boson Higgs production at the LHC.

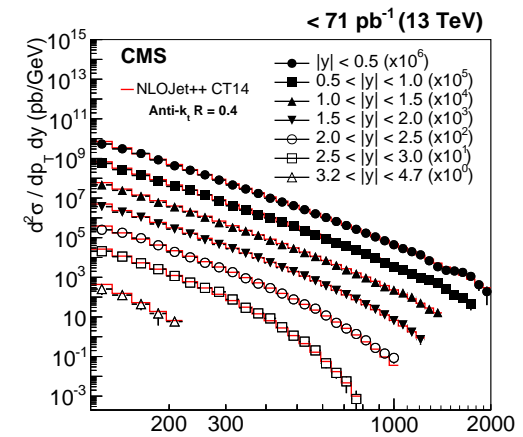

(a) Jet $\mathrm{p}_{\mathrm{T}}(\mathrm{GeV})$
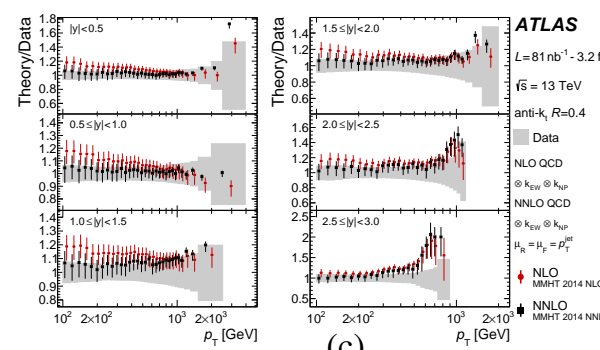

(c)

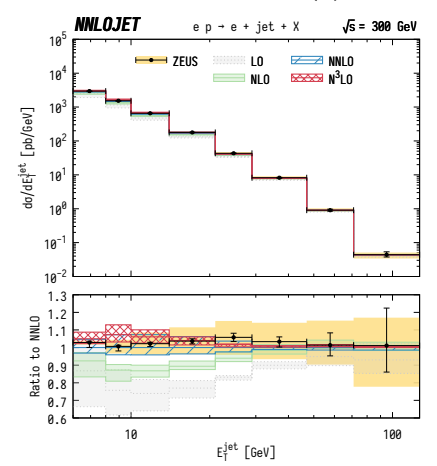

(e)
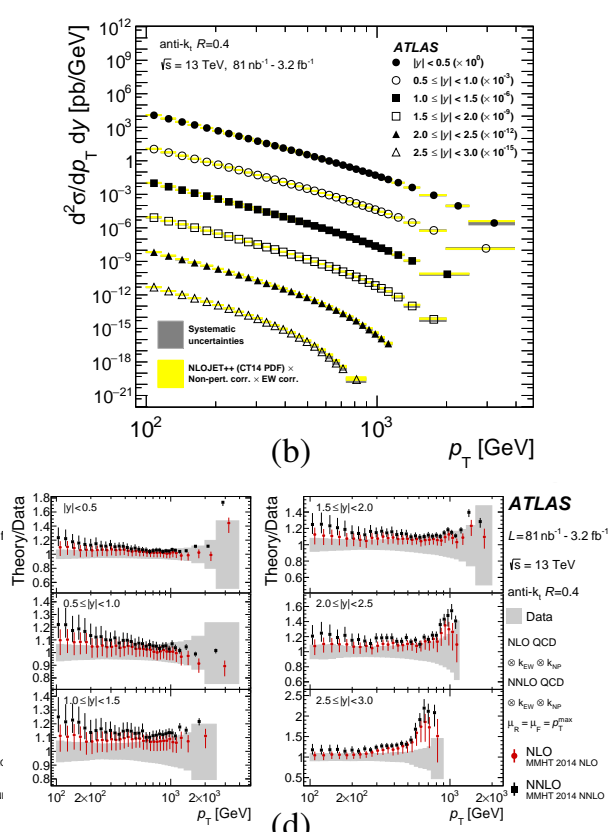

(d)
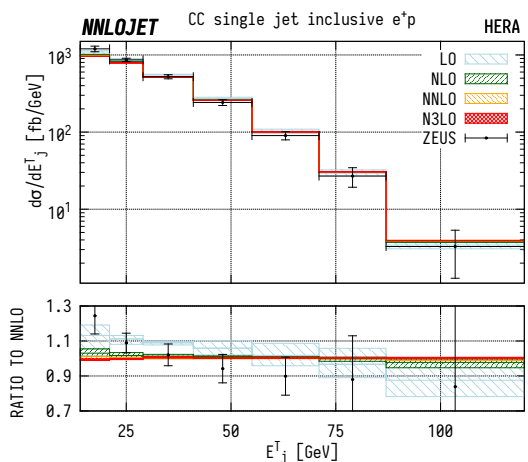

(f)

Figure 1: Inclusive-jet cross sections as functions of $p_{\mathrm{T}}^{\text {jet }}$ in different regions of $y^{\text {jet }}$ from (a) CMS [W] and (b) ATLAS [వ]. The ratio of QCD predictions with (c) $\mu_{R}=p_{\mathrm{T}}^{\text {jet }}$ and (d) $\mu_{R}=p_{\mathrm{T}}^{\max }$ and ATLAS data [D]. Inclusive-jet cross sections as functions of $p_{\mathrm{T}}^{\text {jet }}$ in (e) NC DIS [B] and (f) CC DIS [䧃] from ZEUS compared with pQCD calculations up to $\mathrm{N}^{3} \mathrm{LO}$ [1, 目]. 


\section{Probing QCD with heavy flavours}

Heavy-flavour production constitutes an stringent test of $\mathrm{pQCD}$ with a natural scale given by the mass of the heavy quark. In charm production in CC DIS, the scale is given by the mass of the charm quark. Measurements of these processes provide constraints on the gluon and strange content of the proton and can be used to test suppression effects. They are also sensitive to the electroweak couplings. ZEUS has measured [D] the charm cross section with electron and positron beams. Figure $\nabla(\mathrm{a})$ and (b) show the electroweak (EW) cross section as a function of $Q^{2}$ for $e^{+}$and $e^{-}$beams, respectively, compared with calculations based on different heavy-flavour schemes and PDFs. The predictions provide a good description of the data; a contribution of $30-50 \%$ to the EW cross section from quark-parton-model processes is predicted.

The CMS Collaboration has measured [ [ $]$ ] the cross section for $W+c$ production in the muon plus $D^{*}$ channel. These cross sections are also sensitive to the gluon and strange content of the proton and the mass of the $W$ can be used as the scale. Different suppression and enhancement strange factors have been probed. Figure $\mathbb{Z}(\mathrm{c})$ shows the fiducial cross section for $W+c$ production compared to the predictions based on different PDFs. There is a good agreement between data and the NLO predictions based on different PDFs, except the one extracted by ATLAS (ATLASepWZ16). The strange quark distribution and the suppression factor obtained by CMS agree with earlier results and do not support the enhancement seen by ATLAS.

Beauty production has been studied by ZEUS in NC DIS in the dimuon channel [Q]. The hard scale is provided by the $b$ mass and since there is no jet requirement, it is possible to access very low transverse momenta of the $b$ quarks. The total cross section as well as the differential cross sections as functions of the transverse momentum and pseudorapidity of the muons were measured. In addition, the difference in azimuth of the muon pair and their distance in the $\eta-\phi$ plane were also measured. The measurements are compared with LO and NLO predictions in Fig.D(d). The NLO predictions describe the shape of the data, but have a lower normalisation.

The study of gluon splitting into a $b \bar{b}$ pair is an useful probe of the effects of the large mass of the $b$ quark. The analysis done by ATLAS [एँ] was performed in the high- $p_{T}$, small-angle regime using $b$-tagged small $R$ jets within large $R$ jets. These processes constitute the main background to $H \rightarrow b \bar{b}$ and other searches with $b$ quarks in the final state. ATLAS has measured several observables, such as the distance between the $b$ pair (see Fig. $\nabla(\mathrm{e})$ ). Predictions from LO Monte Carlo (MC) models are compared to the data; differences between the data and the MC models are observed so that the data have the potential to constrain the $p_{T}$-dependent fragmentation distributions.

$\mathrm{LHCb}$ has measured the fiducial cross section for forward top-pair production in the dilepton channel [W]. These measurements provide tests of the Standard Model (SM) and constraints on the proton PDFs. LHCb has the advantage of a larger acceptance in the forward region than other LHC experiments and so access to regions of phase space with a high contribution from $q \bar{q}$ annihilation and high values of $x$ is obtained. The measured fiducial cross section at particle level is compared with the predictions of several NLO QCD calculations in Fig. $\square(f)$ together with the cross section extrapolated to the parton level of top quarks. The measurement has a precision of $20 \%$ and the predictions are compatible with the data, though there is a tendency to be below the measured value. 


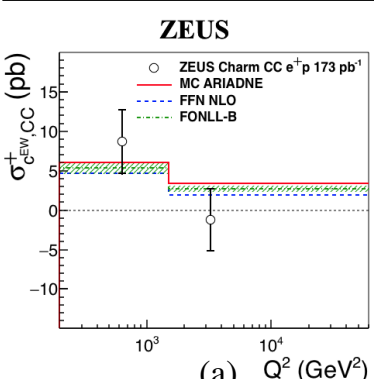

(a) $\mathrm{Q}^{2}\left(\mathrm{GeV}^{2}\right)$

ZEUS preliminary

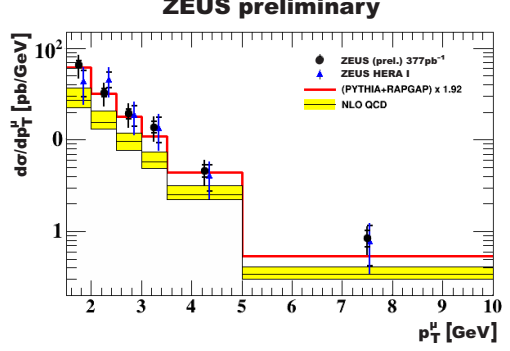

(d)

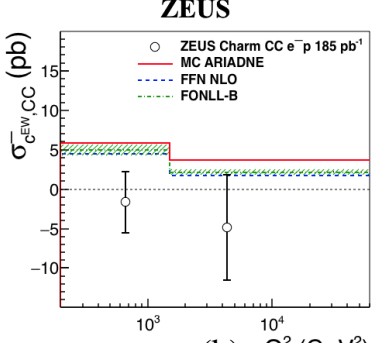

(b) $\mathrm{Q}^{2}\left(\mathrm{GeV}^{2}\right)$

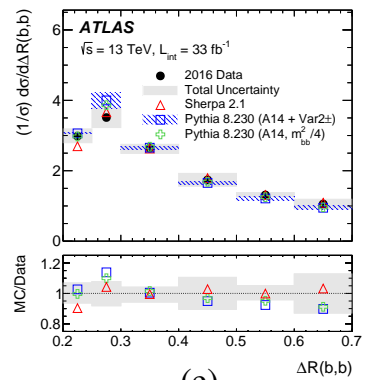

(e)

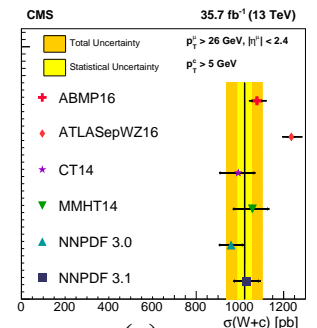

(c)

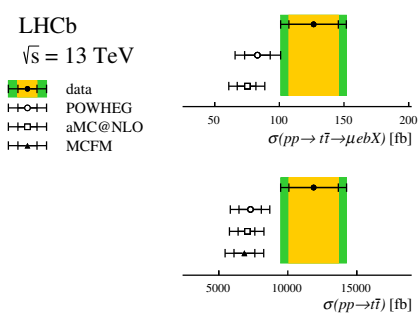

(f)

Figure 2: The ZEUS EW charm cross sections [ [] $]$ in two bins of $Q^{2}$ for (a) $e^{+} p$ and (b) $e^{-} p$ collisions. (c) The CMS inclusive fiducial cross section $\sigma(W+c)$ []. (d) The cross section for dimuon production as a function of the muon transverse momentum from ZEUS [Q]. (e) The $\Delta R(b, b)$ distribution from ATLAS [ए0]. (f) The forward top-pair production cross section from $\mathrm{LHCb}$ [प]].

\section{The hadronic final state}

The hadronic final state can be studied in many processes and with many observables, such as the azimuthal particle correlations measured by ZEUS in DIS []] ]. The correlations for charged hadrons as functions of the multiplicity give information about collective effects in small collision systems such as electron-proton collisions into hadrons and it is possible to obtain limits on the effects inspired by the studies performed in heavy-ion collisions. ZEUS has measured the first and second harmonic coefficients (see Figs. B](a) and (b)) and compared the data and the predictions based on different parton-shower models. No long range correlations are observed and the first harmonic is better described by the MC based on the color-dipole model, whereas the second harmonic is better described by the ME+PS model.

ATLAS has studied jet subtructure observables using groomed jets and comparing the results for different selections [13]], namely dijets, jets from $W$ boson decays and jets from top decays. These studies help to improve the identification of massive particles in those analyses that use boosted topologies. The distributions on the number of subjets in the three samples studied are shown in Fig. B(c); the distributions have a peak at 1, 2 or 3 subjets for dijets, $W$ or top selections, as expected. The predictions from different models differ from the data in some cases and so these measurements can be used to tune the hadronisation models.

LHCb has studied the properties of hadronisation in Z-tagged jets events by means of longitudinal and transverse observables for charged hadrons inside jets [U4]]. This sample is expected to be enriched in light jets and the comparison with other samples enriched in gluon jets provides an opportunity to study the differences in hadronisation using data alone. Figure B(d) shows the distribution of the radial profile of hadrons with respect to the jet axis in three bins of $p_{\mathrm{T}}^{\text {jet }}$. The radial profile $r$ is largely independent of $p_{\mathrm{T}}^{\text {jet }}$ for values of $r$ away from the jet axis. 
CMS has measured the jet-mass cross sections in dijet events with and without grooming. These measurements are sensitive to the internal structure and evolution of jets directly beyond LO. The behaviour of the jet-mass distribution is governed by QCD radiation and the grooming procedure provides a means to differentiate between the effects from hard gluon radiation and the soft contribution arising from pile-up and underlying event. The jet-mass cross section for ungroomed and groomed jets is shown in Figs. B(e) and (f). The grooming procedure decreases the value of the jet mass and reduces the sensitivity to the details of the modelling of the soft effects; in addition, the experimental uncertainties decrease. The theoretical predictions including resummation provide a good description of the data within the uncertainties.

Event shapes have been traditionally used to study the hadronic final state without reconstructing jets, notably at $e^{+} e^{-}$colliders. CMS has measured event-shape observable based on jets [ए6]. These observables are sensitive to the details of hadronisation and are useful to tune the MC models since they test the underlying phenomenological models used to describe these effects. In addition, the effects of initial- (ISR) and final-state (FSR) radiation and the models for multiple-parton scattering (MPI) have been tested by comparing the data with predictions in which different components are disabled. The effect of the ISR contribution is large, whereas those for the FSR and the MPI are smaller. Figure B(g) shows the distribution for the total jet broadening. The comparison with different MC models shows that the energy flow in the transverse plane is described well by the string fragmentation and $p_{T}$-ordered shower models, whereas the out of transverse plane flow is described better by the cluster fragmentation and the angular-ordered shower models.

The normalised inclusive 2- and 3-jet cross sections as functions of the difference in azimuth between the two highest- $p_{T}$ jets $\left(\Delta \phi_{12}\right)$ have been measured [ㅍ] by CMS for nearly back-to-back jets in different regions of $p_{T}^{\max }$. These measurements are directly sensitive to higher orders and also test the parton-shower models and resummed calculations. The normalised cross sections are shown in Figs. B(h) and (i); the measured cross sections for 2 jets are larger than for 3 jets and they increase with $p_{T}^{\max }$ at a fixed value of $\Delta \phi_{12}$ in both cases. The predictions are in general in agreement with the measurements, but some differences are observed, which illustrate the importance of improving the models of soft parton radiation accompanying the hard process.

\section{Inelastic and double-parton scattering}

LHCb has measured the fiducial cross section for inelastic interactions using charged particles and then extrapolating to the full phase space [ए]8]. The inelastic cross section is a fundamental quantity in the phenomenology of high-energy hadronic interactions. It is not calculable in pQCD; thus, models on Regge phenomenology are used and predict an increase of the cross section with $\sqrt{s}$ according to a power law. The fiducial cross section measured by $\mathrm{LHCb}$ is $\sigma_{\mathrm{acc}}=62.2 \pm 0.2 \pm 2.5$ $\mathrm{mb}$. The extrapolation to the full phase space was performed using $\mathrm{MC}$ template fits and the contribution from the inelastic cross section obtained is $\sigma_{\text {inel }}=75.4 \pm 3.0 \pm 4.5 \mathrm{mb}$. This value is compared with those obtained from other LHC experiments in Fig. 因(a). This new value is in agreement with previous measurements and confirms the power-law dependence of the inelastic cross section with the centre-of-mass energy.

Double-parton scattering (DPS) has been studied by ATLAS by measuring its contribution to the four-lepton final state using a neural network (NN) to separate the different components based 


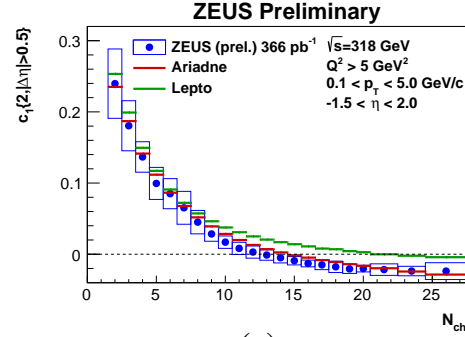

(a)

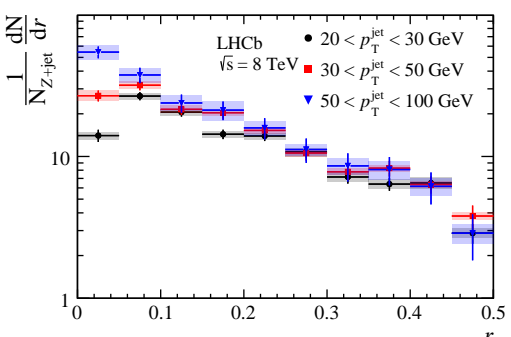

(d)

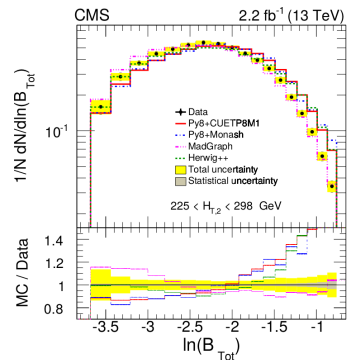

(g)

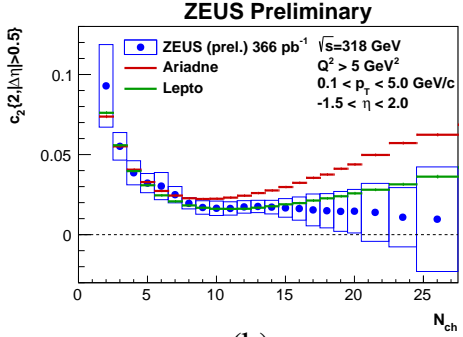

(b)

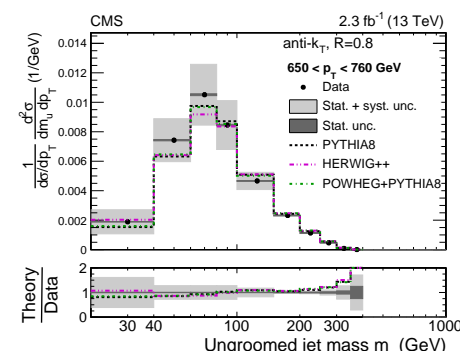

(e)

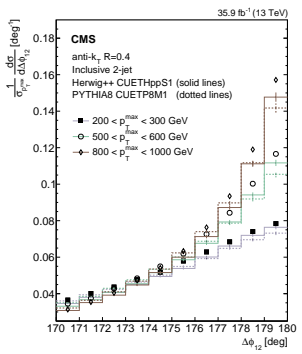

(h)

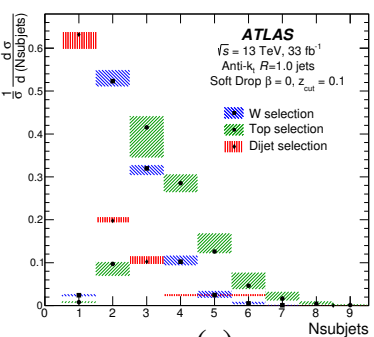

(c)

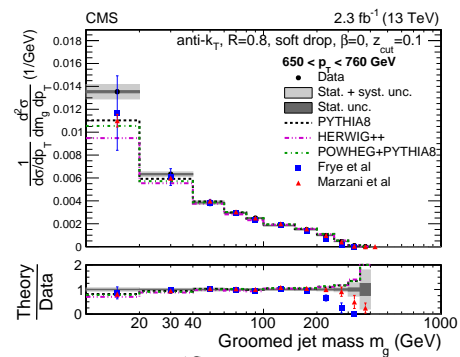

(f)

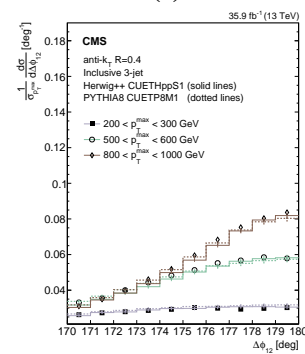

(i)

Figure 3: The $\left\langle\cos \left(n\left(\phi_{\alpha}-\phi_{\beta}\right)\right)\right\rangle$ correlations versus event multiplicity in DIS from ZEUS [ए2] for (a) $n=1$ and (b) $n=2$. (c) Measured subjet multiplicity distributions for dijet, top and $W$ selections from ATLAS [[3]]. (d) Unfolded radial profile distributions of hadrons with respect to the jet axis in three bins of $p_{\mathrm{T}}^{\text {jet }}$ from $\mathrm{LHCb}$ [14]]. Absolute cross section as a function of jet mass for (e) ungroomed and (f) groomed

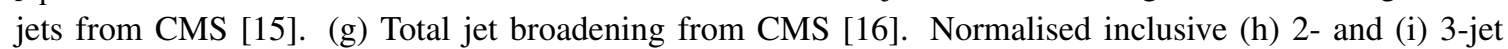
distributions as functions of the azimuthal separation of the two leading jets from CMS [ए]].

on the kinematics of the leptons [[T]. The probability of ocurrence of DPS and the correlations between the decay products are exploited to obtain information on the dynamics. The DPS process is an important background to reactions that also decay into four leptons but proceed via singleparton scattering (SPS), such as $H \rightarrow Z Z$. Figure ब(b) shows the distribution of the NN discriminant used to account for the signal and background. The fraction of DPS is measured to be smaller than 0.042. Since no signal is observed, a lower limit on the effective cross section of $1 \mathrm{mb}$ at $95 \% \mathrm{CL}$ was set, which is consistent with other processes at different centre-of-mass enregies, as shown in Fig. 田(c).

\section{Measuring the fundamental parameter of QCD}

New measurements of $\alpha_{\mathrm{S}}$ are available from ATLAS [20] at NLO and from H1 and ZEUS [R]] at NNLO. The ATLAS determination was extracted from the measurements of azimuthal decorrelations. Measurements of $R_{\Delta \phi}$ as functions of $H_{T}$ in different ranges of $y^{*}$ and for different maximum values of $\Delta \phi$ were performed and compared with LO and NLO QCD calculations; the predictions 


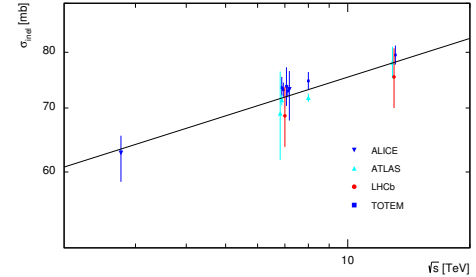

(a)

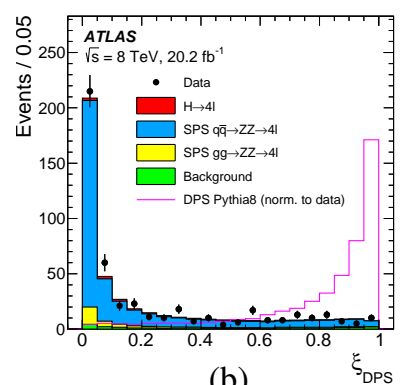

(b)

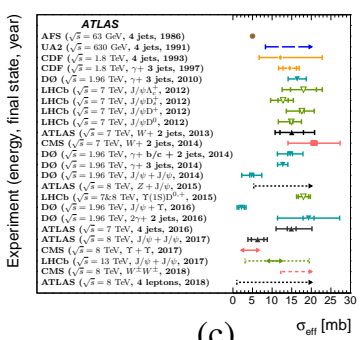

(c)

Figure 4: (a) The total inelastic proton-proton cross section at $\sqrt{s}=2.76,7,8$ and $13 \mathrm{TeV}$ from $\mathrm{LHCb}$ [ए8] and other experiments. (b) The distribution of the output variable of the artificial neural network for data, SPS background and DPS contributions from ATLAS [ष्प]. (c) Summary of measurements and limits on the effective cross section from ATLAS [एप] and other experiments.

give a good description of the data. In some regions of phase space, the theoretical uncertainties are $\approx 5 \%$ and, thus, this constitutes an stringent test of $\mathrm{pQCD}$. The value of the coupling determined at NLO from these measurements is $\alpha_{\mathrm{s}}\left(m_{\mathrm{Z}}\right)=0.1127_{-0.0027}^{+0.0063}=0.1127_{-0.0018}^{+0.0019}$ (exp.) ${ }_{-0.0020}^{+0.0060}$ (theor.). There is a reduction of the uncertainties with respect to other determinations from colliders, especially those of experimental nature, by using this ratio of observables. The scale evolution of the coupling was tested up to values of $1.7 \mathrm{TeV}$, as shown in Fig. 5(a). This new determination of $\alpha_{\mathrm{s}}$ is in good agreement with those from other processes (see Fig. W(b)).

The determination of $\alpha_{\mathrm{s}}$ from H1 and ZEUS is provided by the simultaneous determination of the PDFs and $\alpha_{\mathrm{s}}$ from NNLO QCD fits to inclusive DIS, inclusive-jet and dijet data from H1 and ZEUS. The jet data provides a constraint on the gluon PDF and the use of NNLO jet predictions reduces significantly the uncertainty due to the scale with respect to the NLO analysis. The predictions from HERAPDF2.0Jets NNLO (prel.) agree very well with all data on jet production used as input to the fit; an example of the comparison between data and theory is shown in Fig. [)(c). The value of the coupling obtained is $\alpha_{\mathrm{s}}\left(m_{\mathrm{Z}}\right)=0.1150 \pm 0.0008$ (exp.) ${ }_{-0.0005}^{+0.0002}$ (model) \pm 0.0006 (hadr.) \pm 0.0027 (scale), which is compatible with the world average and competitive with other determinations.

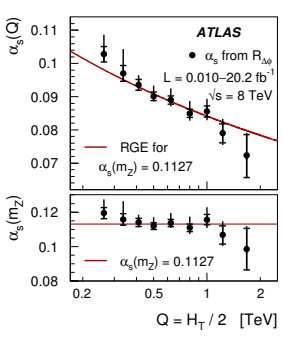

(a)

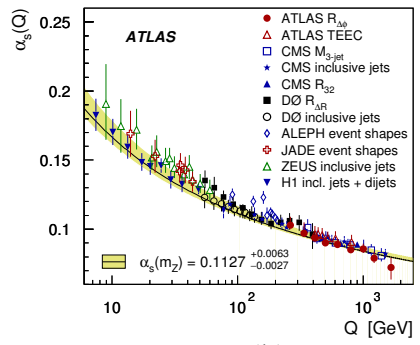

(b)

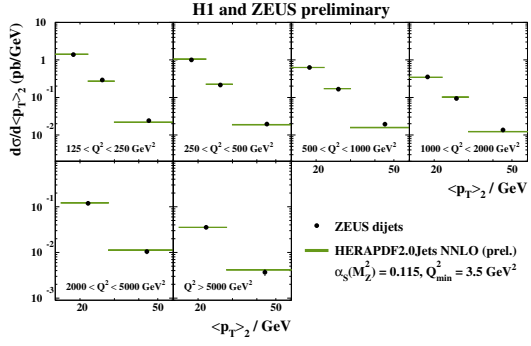

(c)

Figure 5: (a) The $\alpha_{\mathrm{s}}$ results determined from the azimuthal correlations in the range of $262<Q<1675 \mathrm{GeV}$ by ATLAS [EO]]. (b) The $\alpha_{\mathrm{S}}(Q)$ results from ATLAS [20] and other experiments, together with the prediction from the renormalisation group equation. (c) The measured differential dijet cross sections as functions of $\left\langle p_{T}\right\rangle$ in different regions of $Q^{2}$ compared with the NNLO predictions based on the HERAPDF2.0Jets NNLO (prel.) PDF [R]]. 


\section{Vector bosons}

CMS has measured the differential cross sections for isolated photons as functions of photon transverse energy in different regions of photon rapidity with and without a requirement on jets [22]. These measurements test $\mathrm{pQCD}$ with a hard colorless probe and are sensitive to the gluon density in the proton due to the diagrams with highest contribution $(q g \rightarrow \gamma q)$. Figure 6 (a) shows the cross section for inclusive photons. There is a good description of the data by the NLO QCD calculations; there is a potential to constrain further the gluon PDF.

The ratio of isolated-photon cross sections at $\sqrt{s}=13$ and $8 \mathrm{TeV}$ has been performed [D2]] by ATLAS accounting for the correlations of uncertainties, achieving in this way a significant reduction of both the experimental and theoretical uncertainties. This procedure provides a more stringent test of pQCD than with the individual cross sections and a test of the evolution with $\sqrt{s}$. Figure $6(b)$ shows the ratio in a region of photon pseudorapidity; the measured ratio increases with photon transverse energy from $\approx 2$ to above 10 in this region of photon pseudorapidity. The NLO QCD calculations are in good agreement with the measurements. A further reduction of the experimental uncertainties was obtained by measuring the double ratio to the fiducial cross-section ratio for $Z$ production. This procedure cancels out the luminosity uncertainty, which represents a sizeable contribution to the uncertainty in the photon ratio. These tests validate the evolution of isolated-photon production from 8 to $13 \mathrm{TeV}$.

A very recent development has been the computation of isolated-photon cross sections at NNLO [24]. As an example, Fig. 6(c) shows the comparison of the predictions at different fixed orders with the measurement of the cross section as a function of $p_{\mathrm{T}}^{\text {jet }}$ in photon+jet processes from ATLAS [25]. A sizeable reduction of the scale uncertainty is obtained in the NNLO predictions and a significant improvement in the description of the data is provided by the new calculations.

CDF has measured the jet cross sections in association with a $W$ boson [26]. This process constitutes an important background to SM Higgs and top production and searches beyond the SM. Thus, a proper modelling of these processes at lower $\sqrt{s}$ than at LHC is crucial to enhance the physics reach of the LHC studies. CDF has measured the jet multiplicity (see Fig. 6(d)) and the cross section as a function of the jet transverse energy. Also, a ratio of the jet cross section for $N$ jets and that of $N-1$ jets was measured as a function of jet multiplicity. The NLO calculations give a good description of the data. The theoretical uncertainties are dominated by higher orders and comparable in size to those of experimental nature.

ATLAS has measured the production of $W$ and $Z$ bosons at $\sqrt{s}=5.02 \mathrm{TeV}$ in the leptonic channel [D]]. These measurements test both QCD and EW theories and constitute a reference measurement for heavy-boson production in heavy-ion collisions. In particular, the rapidity distributions are sensitive to the underlying dynamics and the PDFs. The differential cross section for $W^{+}$bosons as a function of the lepton rapidity is shown in Fig. G(e). The comparison of the measurements and the NNLO predictions shows that those predictions based on the NNPDF3.1 PDF describe best the data.

CMS has measured the production of $Z Z+$ jets in the four-lepton channel [L8]. These measurements are crucial since the vector-boson fusion ZZjetjet process is a key process to understand the non-abelian gauge structure and the EW symmetry breaking. Since the production cross section of the ZZjetjet process is very low within the SM, it is important to test the QCD corrections to 
vector-boson pair production in association with jets. Figure G(f) shows the measurement of the jet multiplicity at $\sqrt{s}=13 \mathrm{TeV}$. The NLO predictions are in good agreement with the data when the calculations are interfaced with the parton shower simulation of PYTHIA.

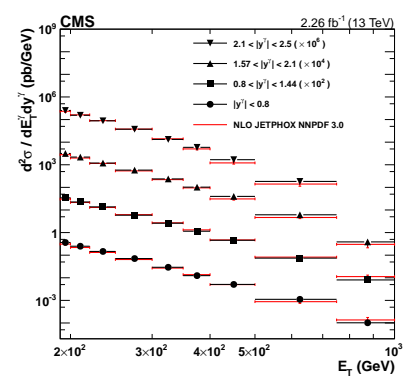

(a)

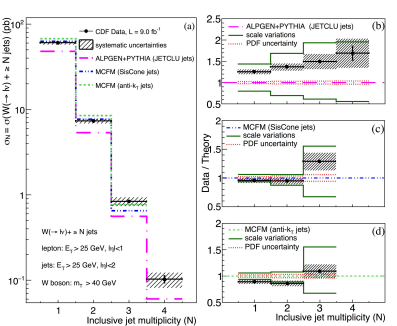

(d)

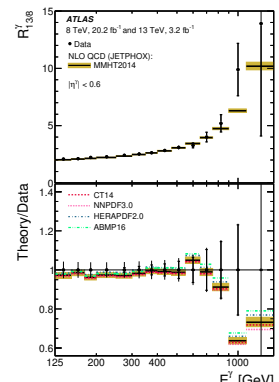

(b)

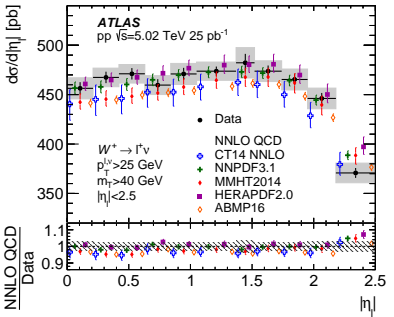

(e)

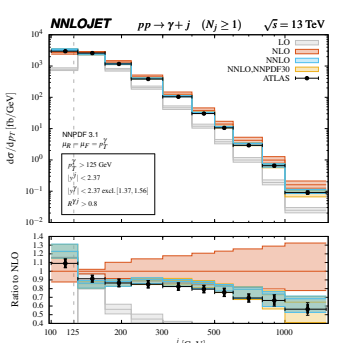

(c)

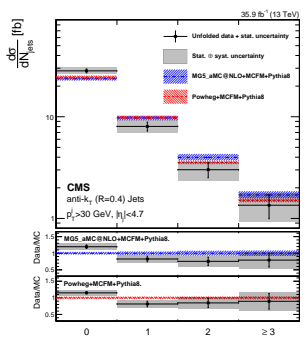

(f)

Figure 6: (a) Differential cross sections for isolated-photon production from CMS [D2]. (b) The ratio of isolated-photon cross sections at $\sqrt{s}=13$ and $8 \mathrm{TeV}$ from ATLAS [23]. (c) Transverse momentum distribution of the leading jet in photon-plus-jet events at LO, NLO and NNLO [24] compared to ATLAS data [ㄷ]. (d) Inclusive jet cross section as a function of the jet multiplicity for $W+$ jets from CDF [26]. (e) Differential cross section for $W^{+}$-boson production as a function of absolute decay lepton pseudorapidity from ATLAS [2]]. (f) Differential cross section for $Z Z$ production as a function of jet multiplicity for $\sqrt{s}=13 \mathrm{TeV}$ from CMS [L8]].

\section{Summary}

There is a wealth of new measurements from HERA, TevaTron and LHC which test QCD with high precision, at the highest energies and at the highest orders available. New measurements which probe the heavy-flavour content of the proton are also available. The hadronic final state is tested now with sophiscated observables based on event shapes and jet subtructure. There are also new precise measurements of the strong coupling and new successful tests of QCD with photons and heavy bosons. From the theoretical side, there has also been considerable progress with the recent publication of new resummed calculations and NNLO and $\mathrm{N}^{3} \mathrm{LO}$ predictions for a variety of processes.

\section{Acknowledgments}

I would like to thank the organisers of the DIS2019 conference for providing me with the opportunity of giving this talk and for a well organised conference. I would like to thank I. Abt, E. 
Barberis, O. Lupton, B. Malaescu, A. Pilkington, M. Ruspa, S. Schmitt, J. Terrón and K. Wichmann for their help in preparing the talk.

\section{References}

[1] CMS Collaboration, "Measurement of the double-differential inclusive jet cross section in proton-proton collisions at $\sqrt{s}=13$ TeV", Eur. Phys. J. C 76 (2016) 451 and R. Zlebcik and C. Young, these proceedings.

[2] ATLAS Collaboration, "Measurement of inclusive jet and dijet cross-sections in proton-proton collisions at $\sqrt{s}=13 \mathrm{TeV}$ with the ATLAS detector", JHEP 1805 (2018) 195 and R. Zlebcik and C. Young, these proceedings.

[3] ZEUS Collaboration, "Forward jet production in deep inelastic ep scattering and low- $x$ parton dynamics at HERA", Phys. Lett. B 632 (2006) 13.

[4] ZEUS Collaboration, "Multi-jet cross-sections in charged current $e^{ \pm} p$ scattering at HERA", Phys. Rev. D 78 (2008) 032004.

[5] J. Currie, T. Gehrmann, E.W.N. Glover, A. Huss, J. Niehues, A. Vogt, "N ${ }^{3}$ LO corrections to jet production in deep inelastic scattering using the Projection-to-Born method", JHEP 1805 (2018) 209 and A. Huss, these proceedings.

[6] T. Gehrmann, A. Huss, J. Niehues, A. Vogt, D.M. Walker, "Jet production in charged-current deep-inelastic scattering to third order in QCD”, Phys. Lett. B 792 (2019) 182 and A. Huss, these proceedings.

[7] ZEUS Collaboration, "Charm production in charged current deep inelastic scattering at HERA", JHEP 1905 (2019) 201 and J. Nam, these proceedings.

[8] CMS Collaboration, "Measurement of associated production of a $W$ boson and a charm quark in proton-proton collisions at $\sqrt{s}=13$ TeV", Eur. Phys. J. C 79 (2019) 269 and I. Josa, these proceedings.

[9] ZEUS Collaboration, "Beauty production in NC $e^{ \pm} p$ DIS”, ZEUS-prel-18-006 and A. Geiser, these proceedings.

[10] ATLAS Collaboration, "Properties of $g \rightarrow b \bar{b}$ at small opening angles in $p p$ collisions with the ATLAS detector at $\sqrt{s}=13$ TeV”, Phys. Rev. D 99 (2019) 052004 and A. Vaidya, these proceedings.

[11] LHCb Collaboration, "Measurement of forward top pair production in the dilepton channel in $p p$ collisions at $\sqrt{s}=13$ TeV”, JHEP 1808 (2018) 174.

[12] ZEUS Collaboration, "Azimuthal particle correlations as a probe of collectivity in deep inelastic electron-proton collisions at HERA", https://inspirehep.net/record/1677149 and I. Abt, these proceedings.

[13] ATLAS Collaboration, "Measurement of jet-substructure observables in top quark, $W$ boson and light jet production in proton-proton $\sqrt{s}=13 \mathrm{TeV}$ with the ATLAS detector", arXiv:1903.02942 and A. Vaidya, these proceedings.

[14] LHCb Collaboration, "Measurement of charged hadron production in Z-tagged jets in proton-proton collisions at $\sqrt{s}=8 \mathrm{TeV}$ ", arXiv:1904.08878.

[15] CMS Collaboration, "Measurements of the differential jet cross section as a function of the jet mass in dijet events from proton-proton collisions at $\sqrt{s}=13$ TeV", JHEP 1811 (2018) 113 and R. Zlebcik, these proceedings. 
[16] CMS Collaboration, "Event shape variables measured using multijet final states in proton-proton collisions at $\sqrt{s}=13$ TeV”, JHEP 1812 (2018) 117.

[17] CMS Collaboration, "Azimuthal separation in nearly back-to-back jet topologies in inclusive 2- and 3-jet events in $p p$ collisions at $\sqrt{s}=13 \mathrm{TeV}$ ", arXiv:1902.04374 and R. Zlebcik, these proceedings.

[18] LHCb Collaboration, "Measurement of the inelastic pp cross-section at a centre-of-mass energy of 13 TeV”, JHEP 1806 (2018) 100 and M. Kucharczyk, these proceedings.

[19] ATLAS Collaboration, "Study of the hard double-parton scattering contribution to inclusive four-lepton production in $p p$ collisions at $\sqrt{s}=8 \mathrm{TeV}$ with the ATLAS detector", Phys. Lett. B 790 (2019) 595 and A. Lennart, these proceedings.

[20] ATLAS Collaboration, "Measurement of dijet azimuthal decorrelations in $p p$ collisions at $\sqrt{s}=8 \mathrm{TeV}$ with the ATLAS detector and determination of the strong coupling”, Phys. Rev. D 98 (2018) 092004 and Z. Hubacek, these proceedings.

[21] H1 and ZEUS Collaborations, "HERAPDF2.0Jets NNLO (prel.), the completion of the HERAPDF2.0 family", H1prelim-19-041 and ZEUS-prel-19-001 and A. Sarkar, these proceedings.

[22] CMS Collaboration, "Measurement of differential cross sections for inclusive isolated-photon and photon+jets production in proton-proton collisions at $\sqrt{s}=13 \mathrm{TeV}$ ", EPJ C 79 (2019) 20 and I. Josa, these proceedings.

[23] ATLAS Collaboration, "Measurement of the ratio of cross sections for inclusive isolated-photon production in $p p$ collisions at $\sqrt{s}=13$ and $8 \mathrm{TeV}$ with the ATLAS detector", JHEP 1904 (2019) 093 and A. Cueto, these proceedings.

[24] X. Chen, T. Gehrmann, N. Glover, M. Höfer and A, Huss, "Isolated photon and photon+jet production at NNLO QCD accuracy”, arXiv:1904.01044 and A. Huss, these proceedings.

[25] ATLAS Collaboration, "Measurement of the cross section for isolated-photon plus jet production in $p p$ collisions at $\sqrt{s}=13 \mathrm{TeV}$ using the ATLAS detector", Phys. Lett. B 780 (2018) 578.

[26] CDF Collaboration, "Measurement of the differential cross sections for $W$-boson production in association with jets $p \bar{p}$ collisions at $\sqrt{s}=1.96$ TeV", Phys. Rev. D 98 (2018) 112005.

[27] ATLAS Collaboration, "Measurements of $W$ and $Z$ boson production in $p p$ collisions at $\sqrt{s}=5.02 \mathrm{TeV}$ with the ATLAS detector", Eur. Phys. J. C 79 (2019) 128 and A. Trofymov, these proceedings.

[28] CMS Collaboration, "Measurement of differential cross sections for $Z$ boson pair production in association with jets at $\sqrt{s}=8$ and 13 TeV", Phys. Lett. B 789 (2019) 19 and P. Govoni, these proceedings. 